\title{
Comparison between Sesame Oil and Glycyrrhizaglabra Effect as Phytoestrogen on Male Albino Rats \\ Eman G.E. Helal ${ }^{1}$,Mohamed A. El-Aleem ${ }^{2}$,Shaimaa S. Ahmed ${ }^{1}$ \\ Zoology Department, Faculty of Science, Al-Azhar University (Girls),Cairo,Egypt. ${ }^{1}$ \\ Physiology Department, Faculty of Medicine, Al-Azhar University,Cairo, Egypt ${ }^{2}$ \\ *Corresponding Author: Eman G.E. Helal, E-mail:emanhelal@ hotmail.com, Mobile: 00201001025364, \\ Orchid.org/0000-0003-0527-7028
}

\begin{abstract}
Background: Phytoestrogen is a plant-derived compound, which has estrogenic effect and it is found in liquorice root extract and sesame oil.Aim of the work:To investigate some biochemical effects of liquorice root extract and sesame oil on male albino rats.
\end{abstract}

Materials and methods: 18 animals were divided randomly into three groups. Group A: Control group, group B: rats treated with oral dose of liquorice $10 \mathrm{mg} / \mathrm{kg}$ body weight/day for one month, andgroup C: rats treated with oral dose of sesame oil $1 \mathrm{ml} / \mathrm{kg}$ body weight/day. At the end of the experiment, blood samples were collected for biochemical analysis.

Results: Liquorice and sesame oil induced highly significant decrease in TC, TG, LDL, VLDL, LDL/HDL $(p<0.01)$ and significant increase in $\operatorname{HDL}(\mathrm{p}<0.05)$. They also showedhighly significantly decrease in $\mathrm{FSH}$, testosterone and sperm countcompared to control group $(\mathrm{p}<0.01)$.

Conclusion:The study supports that the high level intake of liquoriceroot extract orsesame oil caused hormonal disturbance and decreases sperm count.

Keywords:Liquoriceroot, Sesame oil, Lipid profile, Albino rats, Physiological parameters.

\section{INTRODUCTION}

Glycyrrhizaglabra, a family of Leguminosae, is a plant that grows in Egypt and other countries of the world. Its roots possess some nutritional value and medicinal properties. It is widely used as a cold drink and in the preparation of some pharmaceuticals such as hematinic pills ${ }^{(1)}$.

Phytochemical analysis of Glycyrrhizaglabra(liquorice) root extract showedthat it contains saponintriterpenes (glycyrrhizin, glycyrrhetinic acid andliquirtic acid), flavonoids (liquiritin, isoflavonoids and formononetin) andother constituents such as coumarins, sugars, amino acids, tannins,starch, choline, phytosterols and bitter principles, which are most likely responsible for its therapeutic properties $^{(2)}$.

There are many phenols present in liquorice extracts such as liquiritigenin, liquiritin, isoliquiritigenin, isoliquiritin, glabridin and formononetin, which are responsible for the plant estrogen activity of this plant, extracts ${ }^{(3)}$.

Medicinally its root is used widely either as tincture or fluid extract or ingredient in over the counter pharmaceutical products for cough, expectorant, asthma, peptic ulcers and hepaticprotective products.This is because of its antiinflammatory, anti-ulcerative demulcent, antimicrobial and hepatic-protective activities ${ }^{(\mathbf{4})}$.In Japan, intravenously, liquorice components are used for treating hepatitis B and C (National Center for Complementary and Integrative Health, 2016). Other indications still under research like different cancer conditions (Colon, Breast, Hepatic and Prostate),

anti-diabetic, antiangiogenic, neuro-protective, rheumatoid arthritis and cardio-protective ${ }^{(4)}$.

\section{Sesame oil}

Sesame belongs to the family Pedaliaceae. It is one of the richest food source oflignans, amajor type of phytoestrogens known to man since the dawn of civilization ${ }^{(5)}$ and is increasingly incorporated into human diets because ofits health benefits ${ }^{(\boldsymbol{6})}$. Sesame lignans such as sesamin, episesamin, sesamolin, and sesamol isolated from Sesamumindicumseeds are implicated as having certain properties such as antitumorigenic ${ }^{(7)}$ and antioxidant ${ }^{(8)}$. The proximate analysis of sesame seed indicatesthat it contains about 50-60 \% oil, $8 \%$ protein, $5.8 \%$ water, $3.2 \%$ crude fiber, $18 \%$ carbohydrate and $5.7 \%$ ash.It is veryrich in minerals such as calcium, phosphorus and vitamin $\mathrm{E}^{(\mathbf{9 )}}$.The seed oil combats some health conditions like cold and chronic cough and in turn prevents bronchial lung disease. In addition, it helps in improving the blood glucose, glycosylated hemoglobin and lipid-peroxidation ${ }^{(\mathbf{1 0})}$.Besides to its broad use in cooking, the oil is also used in the manufacture of margarine and some pharmaceuticals ${ }^{(\mathbf{1 1})}$. Sesamin, the most abundant lignan in sesame oil ${ }^{\mathbf{( 1 2 )}}$ is known to contain unique amounts of phytoestrogen, and has some effects on sex physiology ${ }^{(12)}$. 


\section{MATERIALS AND METHODS}

Roots of liquorice plant were purchased from market of Agricultural Seeds and Medicinal Plants, Cairo, Egypt. Clean roots (10gm) were soaked in cup of water over night and then filtered. This extract was freshly prepared every day.

Sesame oil extracts were purchased from Cap Pharm for Extracting Natural Oils and Herbs, Cairo, Egypt.

\section{Experimental animal}

The experiment was carried out on 18 malealbino rats Rattusrattus strain weighing (130-140gm) obtained from animal farm of El-Nile Company for Pharmaceutical Products, Cairo, Egypt.Animalswere housed in metallic cages and maintained under standard condition of temperature, humidity and natural. light/dark cycle along the experimental period. Food and water were available throughout the experimentad libitum. Rats were left to acclimatize for one week before starting the experiment.

\section{Experimental design}

Rats were divided into three equal groups (6 rats in each group) as the following:

Group I (control group): maintained on standard pellet diet and tap water ad libitum for 30 days.

Group 2: rats received orally sesame oil $(1 \mathrm{ml} / \mathrm{kg}$ body weight/day) for 30 days.

Group 3: rats received orally liquorice(Glycyrrhizaglabra)rootaquasextract(10 $\mathrm{mg} / \mathrm{kg}$ body weight/day) for 30 days.

\section{Body weight measurement}

Body weight was recorded onzero time and at the end of the experiment.

\section{Blood sample collection}

At the end of the experimental period, the blood samples were collected from the retro-orbital sinus after overnight fasting and the rats were anesthetized by ether. Serum was separatedby centrifugation at $2500 \mathrm{~g}$ for 15 minutes at room temperature to estimate biochemical parameters.

\section{Biochemical analysis}

Assessment of biochemical parameters:

In the present study, total protein (TP) and albumin concentration were estimated, then serum globulin concentrations were calculated according to the formula: Globulin $(\mathrm{g} / \mathrm{dl})=$ total protein $(\mathrm{g} / \mathrm{dl})-$ albumin (g/dl).Aspartate aminotransferase (AST), alanineaminotransferase (ALT), creatinine, BUN andblood glucoseconcentrations as well as lipid profile including total cholesterol, triglycerides and high-densitylipoprotein cholesterol (HDL-C) were alsodetermined. All parameters were estimated using BioMerieux SA kits, France.

Albumin/globulin ratio wasdetermined. In addition, ratios of TC/HDL (risk factor1) and LDL/HDL (risk factor 2) were also calculated after calculation of serum LDL-C (low-densitylipoprotein cholesterol) and VLDL (very low density lipoprotein cholesterol) using the Friedwald's ${ }^{(13)}$ and Norbert ${ }^{(14)}$ formulas, respectivelyas following:

Friedewald's equation:

$\mathrm{LDL}(\mathrm{mg} / \mathrm{dl})=\mathrm{TC}-\{\mathrm{HDL}+[\mathrm{TG} / 5]\}$.

Norbert equation: $\mathrm{VLDL}=\mathrm{TG} / 5$.

TC/HDL (risk factor1)

LDL/HDL (risk factor 2)

\section{Hormonal assay}

Estimation of serum luteinizing hormone (LH), follicles-stimulating hormone (FSH) and testosterone $(\mathrm{T})$ levels by following manufacture instructions of kit. All kits used for hormone assay were MonobindInc.,lake forest CA 92630, USA).

\section{Sperm collection and evaluation}

The left caudal epididymis was separated and the total recovered sperm during $4 \mathrm{~h}$ of incubation in normal saline (volume $=1 \mathrm{ml}, \quad 35 \sim 37^{\circ} \mathrm{C}$ ) was calculated. The sperm concentration was determined by the conventional method using a hemocytometer chamber for the red blood cell count ${ }^{(\mathbf{1 5})}$.

\section{Ethical approved}

The study was approved by the Ethics Board of Al-Azhar University.

\section{Statistical analysis}

The results were expressed as mean \pm SEM(stander error of the mean). Data were analyzed by T-test and were performed using the Statistical Package (SPSS) program, version 19.The Bonferroni test was used as a method to compare significance between groups.

\section{RESULTS}

\section{Body weight and glucose level}

Results of the present study showed a significant increase $(\mathrm{p}<0.05)$ in body weight in the treated groups and non-significantchange in glucose level in the treated groups when compared to control rats (Table 1). 
Table(1):Percentagechanges in body weight and glucose levelin control, sesame oil and liquorice groups.

\begin{tabular}{|l|l|l|l|}
\hline Group & Control & Liquorice & Sesame oil \\
\hline Body weight & $137.6 \pm 0.4$ & $190.6 \pm 0.5 *$ & $195.1 \pm 0.4^{*}$ \\
& & $38.5 \%$ & $41.8 \%$ \\
\hline FBS (mg/dl) & $94.0 \pm 2.8$ & $88.3 \pm 2.0$ & $90 \pm 1.9$ \\
& & $-6.6 \%$ & $-4.2 \%$ \\
\hline
\end{tabular}

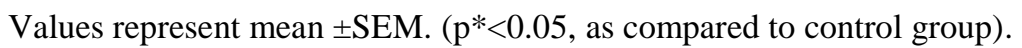

\section{Kidney functions}

The data in table (2) showed non-significantchange in urea and serum creatinine in liquoriceand sesame groups as compared to control (Table 2).

Table(2): Changes in the BUN and creatinine levels in the control,liquoriceand sesame groups.

\begin{tabular}{|l|l|l|l|}
\hline $\begin{array}{l}\text { Groups } \\
\text { Parameters }\end{array}$ & Control & $\begin{array}{l}\text { Liquorice } \\
\text { Extract }\end{array}$ & Sesame oil \\
\hline BUN (mg/dl) & $20.3 \pm 0.2$ & $\mathbf{1 6 . 6 \pm 0 . 4}$ & $\mathbf{2 0 . 1} \pm 0.2$ \\
& & $-\mathbf{1 8 . 2 \%}$ & $\mathbf{- 0 . 9 \%}$ \\
\hline Creatinine (mg/dl) & $0.7 \pm 0.2$ & $\mathbf{0 . 6} \pm 0.01$ & $\mathbf{0 . 6 \pm 0 . 0 0 1}$ \\
& & $-28.5 \%$ & $14.3 \%$ \\
\hline
\end{tabular}

Values represent mean \pm SEM.

\section{Liver functions}

Results of the present study showednon-significantchange in ALAT and ASAT in the liquoriceand sesame groups when compared to control rats (Table 3).

Table (3): Changes in ALAT and ASAT in the control, liquoriceand sesame groups.

\begin{tabular}{|l|l|l|l|}
\hline Groups & Control & $\begin{array}{l}\text { Liquorice } \\
\text { extract }\end{array}$ & Sesame oil \\
\hline Parameters & & $20.0 \pm 0.4$ & $18.9 \pm 0.3$ \\
& $22.5 \pm 0.6$ & $-11.1 \%$ & $16 \%$ \\
\hline ASAT(U/L) & $33.5 \pm 0.5$ & $29.1 \pm 0.3$ & $32.1 \pm 0.2$ \\
& & $-13.1 \%$ & $4.2 \%$ \\
\hline
\end{tabular}

Values represent mean $\pm \mathrm{SE}$ (stander error).

\section{Lipid profile}

Results of the present study showed that liquoriceand sesame produced highly significant decrease $(\mathrm{p}<0.01)$ in TC,TG, LDL, VLDL and LDL/HDLwhen compared to control group. While, there was highly significant increase ( $\mathrm{p}<0.05)$ in HDL, as compared to control animals (Table 4).

Table (4): Changes in the lipid profile in the control, liquorice andsesame groups.

\begin{tabular}{|c|c|c|c|}
\hline Groups $\quad$ Parameters & Control & $\begin{array}{c}\text { Liquorice } \\
\text { extract }\end{array}$ & Sesame oil \\
\hline T C $(\mathrm{mg} / \mathrm{dl})$ & $141.3 \pm 0.42$ & $\begin{array}{c}86.3 \pm 1.5 * * \\
-38.9 \%\end{array}$ & $\begin{array}{c}85 \pm 0.01 * * * \\
-39.8 \%\end{array}$ \\
\hline T G(mg/dl) & $71.5 \pm 1.56$ & $\begin{array}{c}56.8 \pm 4.4^{*} \\
-20.5 \%\end{array}$ & $\begin{array}{l}43.5 \pm 2.1 * * \\
-39.1 \%\end{array}$ \\
\hline HDL(mg/dl) & $50.3 \pm 0.33$ & $\begin{array}{c}\text { 61.3 } \pm 0.33 * \\
21.8 \%\end{array}$ & $\begin{array}{l}60 \pm 0.01 * \\
19.3 \%\end{array}$ \\
\hline LDL(mg/dl) & $71.2 \pm 1.27$ & $\begin{array}{l}\text { 14.1 } \pm 1.1 * * \\
-80.1 \%\end{array}$ & $\begin{array}{r}16.3 \pm .58 * * \\
-77.1 \% \\
\end{array}$ \\
\hline VLDL(mg/dl) & $14.3 \pm 0.31$ & $\begin{array}{l}11.0 \pm 0.9 * \\
-23.0 \%\end{array}$ & $\begin{array}{c}8.7 \pm 0.58 * * \\
-39.1 \% \\
\end{array}$ \\
\hline LDL/HDL(mg/dl) & $1.22 \pm 0.007$ & $\begin{array}{l}0.22 \pm .01 * * \\
-81.9 \%\end{array}$ & $\begin{array}{r}0.27 \pm 0.01 * * \\
-77.8 \%\end{array}$ \\
\hline TC/HDL(mg/dl) & $2.4 \pm 0.008$ & $\begin{array}{l}1.4 \pm .22 * * \\
-41.6 \%\end{array}$ & $\begin{array}{l}1.4 \pm 0.01 * * \\
41.6 \%\end{array}$ \\
\hline
\end{tabular}

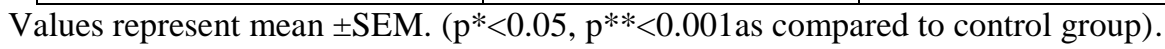




\section{Protein profile}

In the present study, administration of liquoriceand sesame to normal rats showed non-significant change in total protein, albumin, globulin andalbumin/globulin ratio in the treated groups when comparedto control rats (Table 5).

Table (5): Changes in the total protein, albumin, globulin and albumin/globulin levels in control and treated groups.

\begin{tabular}{|c|c|c|c|}
\multicolumn{1}{|l|}{ Parameters } & Control & $\begin{array}{c}\text { Liquorice } \\
\text { extract }\end{array}$ & Sesame oil \\
\hline Total protein (g/dl) & $6.4 \pm 0.12$ & $5.5 \pm 0.20$ & $6.0 \pm 0.1$ \\
\% of change & & $-14.0 \%$ & $-6.25 \%$ \\
\hline Albumin (g/dl) & $3.7 \pm 0.11$ & $4.0 \pm .15$ & $3.5 \pm 0.19$ \\
\% of change & & $8.1 \%$ & $-5.4 \%$ \\
\hline Globulin (g/dl) & $2.7 \pm 0.10$ & $2.3 \pm 0.22$ & $2.5 \pm 0.3$ \\
\% of change & & $-14.8 \%$ & $-7.4 \%$ \\
\hline Albumin/Globulin(g/dl) & $1.3 \pm 0.1$ & $1.7 \pm 0.06$ & $1.4 \pm 0.06$ \\
\% of change & & $30.7 \%$ & $7.6 \%$ \\
\hline
\end{tabular}

Values represent mean \pm SEM.

\section{Hormones}

The present study showed that administration of liquorice extract to normal rats showed a highly significant decreased FSH and testosterone $(\mathrm{p}<0.01)$, while in sesame group there were a significantly decreased FSH and testosterone $(\mathrm{p}<0.05)$ when compared to control rats (Table 6$)$.

Table(6): Changes in FSH, LH and Testosterone levels in control, sesameand liquorice groups.

\begin{tabular}{|l|c|c|c|}
\hline Groups & Control & $\begin{array}{c}\text { Liquorice } \\
\text { extract }\end{array}$ & Sesame oil \\
\hline FSH(ng/ml) & $2.9 \pm 0.1$ & $\begin{array}{c}1.9 \pm 0.1 * * \\
-34.4 \%\end{array}$ & $\begin{array}{c}2.0 \pm 0.1 * \\
\text { \% of change }\end{array}$ \\
\hline LH(ng/ml) & $1.9 \pm 0.1$ & $1.7 \pm 0.1$ & $1.8 \pm 0.1$ \\
\% of change & & $-10.5 \%$ & $-5.2 \%$ \\
\hline Testosterone( $\mu$ u /dl) & $3.9 \pm 0.2$ & $2.8 \pm 0.2 * *$ & $2.9 \pm 0.2 *$ \\
\% of change & & $-39.3 \%$ & $-25.06 \%$ \\
\hline
\end{tabular}

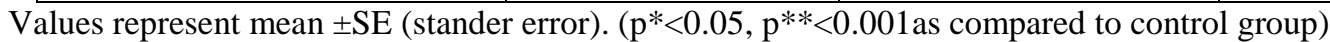

\section{Sperm count}

The data in table (7) showed decrease in sperm count in liquoriceand sesame groups when compared to control rats.

Table (7): Sperm count in the control, sesame and liquorice group.

\begin{tabular}{|l|c|c|c|}
\hline Groups Control & $\begin{array}{c}\text { Liquorice } \\
\text { extract }\end{array}$ & Sesame oil \\
\hline $\begin{array}{l}\text { Sperm count* } * 10 \% \mathrm{ml} \\
\text { \% of change }\end{array}$ & 100 & $\begin{array}{c}38 \pm 0.01 \\
-62 \%\end{array}$ & $\begin{array}{c}63 \pm 0.01 \\
-37 \%\end{array}$ \\
\hline
\end{tabular}

\section{DISCUSSION}

Body weight

The findings in this study showed an increase in body weight in both experimental groups treated with Glycyrrhizaglabra(liquorice)and sesame oil. In Glycyrrhizaglabra the increase in body weight by Glycyrrhizaglabraroot extract is in accordance with that reported by Miller ${ }^{(16)}$ whodemonstrated that Glycyrrhizaglabrainhibits $11 \quad \beta$-hydroxysteroid dehydrogenase and induces excess release of mineralocorticoids, which causes retention of sodium and water that leads to edema and increase in body weight. However, the increased body weight is explained by the improvement in feeding efficiency $^{(17)}$.

While in Sesame oil group the increase in body weight was due tounsaturated fatty acids such as oleic acid and linoleic acid, which increase the effectiveness of non-steroidal hormones such as thyroid and growth hormones which are important 
factors in increasing body weight by stimulating the basal metabolic rate ${ }^{(18)}$.

\section{Lipid profile}

The decrease in serum total cholesterol and triglycerides reported in this study following oral administration of Glycyrrhiza glabra ((liquorice) root for 4 weeks is similar to that reported by Khushbaktovaet al. ${ }^{(19)}$ and Fuhrman et al. ${ }^{(20)}$. The studies of previously mentionedauthors attributed the hypocholesterolemic effect of liquoriceto the presence of certain isoflavones, which act as antioxidants via inhibition of LDLcoxidation which inhibits the local mechanism of atherogenesis. Moreover, Nikitinaet $\boldsymbol{a l l}^{\left({ }^{(21)}\right.}$ reported that the glycosides of Glycyrrhizaglabraprevent accumulation of cholesterol in cells as well as human blood serum. A significant decline in plasma LDL-cholesterol in treated groupscould be correlated with saponin content of liquoriceroot, wheresaponin enhances the hepatic LDL-receptor levels, increase hepatic uptake of LDL-cholesterol and aids its catabolism to bile acid ${ }^{(22)}$. Saponinis known to lower triglyceride by inhibiting pancreatic lipase activity. While, the decline in VLDL cholesterol levels in the treated groupscould be directly correlated to decline in triglyceride levels of these groups, as it is well established that VLDL particles are the main transporters of triglyceride in plasma $^{(23)}$. Phytosterolsare reported to displace intestinal cholesterol and reduce cholesterol absorption from intestine ${ }^{(24)}$. Saponins are capable of precipitating cholesterol from micelles and interfere with enterohepatic circulation of bile acids, making it unavailable for intestinal absorption ${ }^{(25)}$.

Insesame oil group, there was a significant decrease in lipid profile.Sesame lignans (sesamin and/or episesamin) reduced serum and liver cholesterol concentrations by inhibiting absorption and synthesis of cholesterol.Ogawa et al. (26) reported that daily oral intake of sesamin in hypercholesterolemic patients for 4 weeks significantly decreased total cholesterol and LDL-C concentrations. In rat, the mechanism for the hypocholesterolemic effect of sesaminis believed to be related to inhibition of intestinal absorption of cholesterol, increased excretion of cholesterol into bile and decreased activity of 3-hydroxy-3methylglutaryl coenzyme A reductase.

\section{Hormones}

Change in hormonal levels in this study showed a significant reduction in follicle stimulating hormone and testosterone. Testosterone is a steroid hormone from androgen group, and is one of the major sex hormones produced by the body in both men and women. It plays key roles in health and well-being. FSH and testosterone are involved as synergistic in the process ofspermatogenesis ${ }^{(27)}$. Armaniniet al. ${ }^{(28)}$ found that liquorice reduces serum testosterone in healthy men.Alsoliquorice inhibits $6 \beta$-hydroxy steroid dehydrogenase and stimulating aromatase, resulting in reduced serum testosterone. Liquorice reduced the serum testosterone level and affected androgen metabolism by inhibiting the enzyme $\beta-6$ HSD and 17$\beta$ HSDhydroxysteroid dehydrogenase or by stimulating aromatase. Therefore, it was proposed that liquorice causes the deficiency in serum testosterone ${ }^{(\mathbf{2 9})}$, glabridin, the major isoflavone in liquorice and many other phenols such as liquiritigenin, liquiritin, isoliquiritigenin, isoliquiritin, glabridin and formononetinthat are responsible for phytoestrogen activityand estrogenic properties $^{(30)}$, which cause hormonal disturbance and decrease the level of FSH and Testosterone, whichlead to decrease in sperm count.

While in sesame oil group, the decrease in serum FSH and testosteronewas approved by the study of Brown and Chakraborty ${ }^{(31)}$ who showed that estrogen agonist like clomiphene has been found to decrease the synthesis and/or release of gonadotrophins with the implication of both low serum LH and testosterone concentration found in male rats. FSH concentration decreased in a high dose of sesame group compared to control group. Shittuet al. ${ }^{(32)}$ found that sesame phytoestrogen lignanscan stimulate testosterone aromatization to estradiol, or convert it to Dihydrotestosterone.

\section{CONCLUSION}

Therefore, it can be concluded that the decrease in the concentrations of testosteronewasdue to its conversionintoestradiol which is done by aromatase and reductase enzymes and sesame lignans. This studysuppose that the decrease in FSH and testosterone level leads to decreasing in the sperm count.

\section{REFERENCE}

1.Fenwick G,Lutomski J,Nieman C (1990): Glycyrrhizaglabra L. (Liquorice): Composition, uses and analysis. Food Chem., 38(2): 119-143.

2.Arystanova T, IrismetovM,SophekovaA(2001): Chromatographic determination of glycyrrhizinic acid in Glycyrrhizaglabrapreparation. Chem Nat Com., 37: 89-91

3. Tang Z L, TongY, ChenX, ChenX, WangY, LuJ, AngelesL (2015): A systematic review of the anticancer properties of compounds isolated from licorice (Gancao). Planta Med.,18: 1670-1687.

4.PengF, DuQ, PengC, WangN, TangH, XieX, ShenJ, Chen J (2015): A review: The pharmacology of Isoliquiritigenin'.Phytotherapy Research, 29:969-977. 
5.ZavarehM, HoogenboomG, RahimianM H, ArabdA (2008): A decimal code to describe the growth stages of sesame (SesamumorientaleL.). Int J Plant Prod., 2:193-206.

6.ShittuL A J, Shittu R K, Adesite S O, Ajala M O,Bankole, M A, Benebo AS (2008): Sesame radiatumPhytoestrogens Stimulate Spermatogenic Activity and Improve Sperm Quality in Adult Male Sprague Dawley Rat Testis. Int J Morphol., 26(3):643652.

7.HiroseN,InoueT,NishiharaK,SuganoM,AkimotoK,Shi mizuS,Yamada H (1991): Inhibition of cholesterol absorption andsynthesisin rats by sesame. J. Lipid Res., 32:629-638.

8.Jeng KCG, HouRCW (2005):Sesamin and Sesamolin: Nature'sTherapeutic Lignans. CurrEnzymInhib.,1:1120.

9.ObiajunwaEI, Adebiyi FM, OmodePE (2005): Determination of Essential Minerals and Trace Elements in Nigerian Sesame Seeds, Using TXRF Technique. Pakistan J Nutr.,4: 393-395.

10.Kanu PJ (2011): Biochemical analysis of black and white sesame seedsfromChina.AmJBiochemMolBiol.,1:145-157.

11.Sangha M, Gupta P, Tharpo V (2004): Storage studies on plant oils andtheir methyl esters. E Journal, 7:1-15.

12.FukudaY,NagataM,NamikiMJ(1986):ContributionofL ignanAnalogues to Antioxidant Activity of Refined Unroasted Sesame SeedOil.JAmOilChem.Soc., 63:1027-1031.

13.JacklinA, RatledgeC,Welham K,BilkoD, NewtonCJ(2003): Thesesame oil constituent, sesamol, induces growth arrest and apoptosisofcancerandcardiovascularcells.Ann.NewYor kAcadSci., 1010:374-380.

14.Friedwald's WT, Levy RI,Fredrickson DS et al. (1999): Estimation of the concentration of low-density lipoprotein cholesterol in plasma without use of the preparative ultracentrifuge. Clin Chem., 18:499-502.

15.Norbert WT (1995): clinical guide to laboratory tested. $3^{\text {rd }}$ ed.saunders W.B.,Company,Philadelphia.

16. WHO (2010): WHO laboratory manual for the examination and processing of human semen. Fifth ed. Geneva, Switzerland: WHO press: 2244.https://apps.who.int/iris/handle/10665/44261

17.Miller I (1998): Herbal medicine: Selected clinical considerations focusing on known or potential drugherb interaction. Arch. Inter Med., 158: 2200-2211.

18.Alaauldeen S M AL-Sallami(2017): Effect of Sesame Oil on Male Rats Treated with Acrylamide in some Physiological and Hormonal Blood Criteria. IJCPR., 8: 134-140.

19.KhushbaktovaZ, Syrov V, Batirov E (1991): Effect of flavonoids on the course of hyperlipidemia and atherosclerosis under experimental conditions. KhimikoFarmatsevticheskiiZhurnal, 25:54- 57.
20.FuhrmanB, Vaya J, BelinkyP,Aviram M(1999): The isoflavoneglabridin inhibits LDL oxidation. https://link.springer.com/content/pdf/10.1007\%2F3540-27661-0.pdf

21.NikitinaN, KhalilovE, TertovV,OrekhvA (1995): In vitro decrease in the atherogenicity by polyunsaturated phosphotidylcholine.Byu. Eksper. Biol Med., 119: 497501.

22. Harwood J H J, Chandler C E, Pellarin L D, Bangerter F W, Wilkin RW, Long CA(1993): Pharmacologic consequences of cholesterol absorption inhibition: alteration in cholesterol metabolism and reduction in plasma cholesterol concentration induced by the synthetic saponinbetatigogenincellobioside (CP88818; tiqueside). J Lipid Res., 34: 377-95.

23. Howell $\mathbf{T} \mathbf{J}$, MacDougall $\mathbf{D} \mathbf{E}$, Jones $\mathbf{P} \mathbf{J} \quad \mathbf{H}$ (1998): Phytosterols partially explain differences in cholesterol metabolism caused by corn or olive oil feeding. J Lipid Res., 39: 892-900.

24.Ikeda I, Sugano M (1998): Inhibition of cholesterol absorption by plant sterols for mass intervention. CurrOpinLipidol., 9: 527-531.

25.Oakenfull D G, Sidhu G S(1990): Could saponins be a useful treatment for hypercholesterolaemia? Eur J ClinNutr., 44: 79-88

26.Ogawa H, Sasagawa S, Murakami T, Yoshizumi H (1995): Sesame lignans modulate cholesterol metabolism in the stroke-prone spontaneously hypertensive rat. ClinExpPharmacol Physiol., 22: 310312.

27.BerneRM, LevyMN(1993): Physiology. Third ed. St. Louis, Mosby,pp: 950-959.

28.ArmaniniD, Bonanni G, Mattarello M J, Fiore C, Sartorato P, Palermo M (2003): Licorice Consumption and Serum Testosterone in Healthy Man. Endocrinol Diabetes ExpClin., 111: 341-343.

29.TamirS, Eizenberg M, Somjen D, Stern N, Shelach R, Kaye A, Vaya J (2000): Estrogenic and antiproliferative properties of glabridin from licorice in human breast cancer cells.Cancer Res., 60: 5704-5709.

30.TangZ, LiT, TongY, ChenX, Chen X, Wang Y, LuJ, Angeles L (2015): A systematic review of the anticancer properties of compounds isolated from licorice (Gancao). Planta Med.,81:1670-1687.

31.BrownJL,Chakraborty PK (1998): Characterization of the effects of clomiphene citrate on reproductive physiology in male rats of various ages. ActaEndocrinol., 118:437-443.

32.Shittu LAJ, Shittu RK, Adesite SO, AjalaMO,Bankole MA, Benebo AS(2008): Sesame radiatum Phytoestrogens Stimulate Spermatogenic Activity and Improve Sperm Quality in Adult Male Sprague Dawley Rat Testis. Int J Morphol., 26:643652. 